\title{
Influencia de la tecnología en el aprendizaje del estudiante para resolver problemas matemáticos
}

\author{
Carlos Wilson Lizarazo Gómez \\ carlos_wilsonl@hotmail.com \\ Centro de Investigación y Estudios Avanzados \\ Instituto Politécnico Nacional de México.
}

\section{Resumen}

En esta investigación descriptiva, se explora el aprendizaje para la solución de problemas del álgebra con Cabrí y los tres niveles de aprendizaje propuestos por Pluvinage. Es una contribución didáctica para los estudiantes del curso de razonamiento cuantitativo de la Universidad del Atlántico y el Cinvestav IPN de la ciudad de México, para la utilización de herramientas tecnológicas tales como los Software de geometría dinámica los cuales potencian las competencias de los estudiantes en cada uno de los pensamientos que desarrollan en la asignatura de razonamiento cuantitativo. Son las primeras pruebas implementadas en el aprendizaje del área matemática en dichas instituciones, en la que participaron estudiantes de primer semestre de humanidades y música de la Facultad de Educación. Sus edades fluctuaban entre los 17 y 20 años. En el periodo de la experimentación todos estaban cursando la asignatura de razonamiento cuantitativo.

\section{Palabras Clave}

Conjetura; software dinámico; educación; solución de problemas

Recibido 17/03/2020-Aceptado 21/05/2020 
Influence of technology in the teaching-learning processes of mathematics in problem solving.

\begin{abstract}
This article was written as a didactic contribution for the students that have a quantitative reasoning course of the Universidad del Atlántico and the Cinvestav IPN of Mexico City, for the use of technological tools such as Dynamic Geometry Software which enhance the skills of the students in each of the thoughts they develop in the subject of quantitative reasoning. These are the first tests implemented in the learning of the mathematical area in these institutions, in which first semester students of humanities and music of the Faculty of Education participated. Their ages fluctuated between 17 and 20 years. In the period of experimentation, everyone was studying the subject of quantitative reasoning. At the level of this descriptive research, learning to solve algebra problems with Cabrí and the three levels of learning proposed by Pulvinate will be explored.
\end{abstract}

\title{
Keywords
}

Conjecture; dynamic software; education; solved problems 
Tecnologia e sua influência nos processos de ensino-aprendizagem de matemática

\section{Resumo}

Nesta investigação descritiva, o aprendizado para resolver problemas de álgebra com Cabrí e os três níveis de aprendizado propostos pelo Pluvinage são explorados. É uma contribuição didática para os alunos do curso de raciocínio quantitativo da Universidad del Atlántico e do IPN Cinvestav na Cidade do México, para o uso de ferramentas tecnológicas como o Dynamic Geometry Software, que aprimoram as competências dos alunos em cada um dos pensamentos que se desenvolvem no assunto do raciocínio quantitativo. São os primeiros testes implementados no aprendizado da área matemática nessas instituições, nos quais participaram estudantes do primeiro semestre de ciências humanas e música da Faculdade de Educação. Suas idades variaram entre 17 e 20 anos. No período da experimentação, todos estavam discutindo o raciocínio quantitativo.

\section{Palavras chave}

Acho; software dinâmico; Educação; solução de problemas 


\section{Introducción}

Se describe en este artículo el procedimiento utilizado en la investigación, en cuanto a la forma en que fueron tomados los datos. Se proporcionan elementos básicos y necesarios para analizar y describir los razonamientos principales de los alumnos en cuanto al uso de Software dinámico en la solución de problemas de tipo geométrico; indicando el objetivo principal de cada actividad. Las tareas que fueron utilizadas en este estudio y la forma en que fueron abordadas por los alumnos, también constituye un elemento importante de comprensión y análisis en el diseño metodológico. Además de exhibir aspectos relacionados con los sujetos participantes en esta investigación, es de interés documentar los procesos de argumentación de ellos para el análisis global del concepto de solución dado por los alumnos, cuando resuelven un problema matemático con software dinámico.

\subsection{Planteamiento del Problema}

Dado el avance tecnológico, en el ámbito educativo, es posible plantearse preguntas como la siguiente: ¿cómo influye la tecnología en la enseñanza y el aprendizaje de las matemáticas? Para tratar de responder esta pregunta se han realizado varios trabajos relacionados con diversas tecnologías, enfocados a la enseñanza y el aprendizaje de las matemáticas. Por ejemplo, Kieran y Guzmán (2003) afirman que en la investigación sobre el uso de tecnología en el aprendizaje-enseñanza de las matemáticas se está realizando de dos distintas maneras: la primera está relacionada con el diseño de actividades, y la segunda, con el desarrollo de éstas (p. 41). Por otra parte, Verillon y Rabardel (2015) estiman crucial que los profesores comprendan el diseño de actividades y contribuyan al florecimiento de esa sinergia entre el alumno y la tecnología (p. 2). Las afirmaciones anteriormente señaladas enfatizan diversos aspectos relacionados con el uso de herramientas tecnológicas, que permiten analizar interacciones entre alumnos y maestros, cuando son utilizadas; por ejemplo: de la calculadora o la computadora para interpretar por medio de una gráfica el concepto de la primera y segunda derivada en cálculo diferencial, o en la enseñanza del álgebra, cuando se trata de resolver sistemas de ecuaciones lineales con dos incógnitas mediante el uso de software dinámico. Así, en los últimos años se han realizado investigaciones concernientes al uso de 
las herramientas tecnológicas en la enseñanza y aprendizaje de las matemáticas (Mariotti, 2003; Verillon y Rabardel 2015; Guzmán y Kieran; 2002, Kieran y Guzmán 2003; Artigue, 2001; Guin and Trouche, 1999; Lizarazo y García, 2011; Lagrange et al., 2000, entre otros). Guin y Trouche (1999) mencionan, en forma resumida, que el uso de herramientas tecnológicas: "le da a los alumnos la oportunidad de solidificar y ampliar sus conocimientos matemáticos [...] y pueden estimular el aprendizaje de las matemáticas en los alumnos” (p. 705). Sin embargo, Horgan (2013, p. 47) afirma que la comunidad matemática continúa considerando a las computadoras como invasoras, intrusas en el campo de la enseñanza y el aprendizaje de las matemáticas. Sin lugar a dudas, este tipo de opiniones afecta directamente las investigaciones relacionadas con la enseñanza y el aprendizaje de las matemáticas en alumnos de cualquier nivel educativo. Presentación y análisis de la naturaleza del estudio y los desarrollos de los estudiantes, mediante el uso de Software dinámico Es de interés fundamental describir y analizar en esta investigación el concepto de solución que aportan los alumnos cuando resuelve un problema de matemáticas, mediante el uso de Software dinámico.

Así, en los últimos años se han realizado investigaciones concernientes al uso de las herramientas tecnológicas en la enseñanza y aprendizaje de las matemáticas (Mariotti, 2003; Verillon y Rabardel 1995; Guzmán y Kieran; 2002, Kieran y Guzmán 2003; Artigue, 2001; Guin and Trouche, 2014; Lizarazo y García, 2015; Lagrange et al., 2014, entre otros). Guin y Trouche (2014) mencionan, en forma resumida, que el uso de herramientas tecnológicas: "les da a los alumnos la oportunidad de solidificar y ampliar sus conocimientos matemáticos [...] y pueden estimular el aprendizaje de las matemáticas en los alumnos” (p. 705). Sin embargo, Horgan (1993, p. 47) afirma que la comunidad matemática continúa considerando a las computadoras como invasoras, intrusas en el campo de la enseñanza y el aprendizaje de las matemáticas. Sin lugar a dudas, este tipo de opiniones afecta directamente las investigaciones relacionadas con la enseñanza-aprendizaje de las matemáticas en alumnos de cualquier nivel educativo. 


\section{Diseño metodológico}

Investigación de tipo descriptiva en la que participaron 10 alumnos tercer semestre de ingeniería. Sus edades fluctuaban entre los 17 y 20 años. En el periodo de la experimentación todos estaban cursando la asignatura de razonamiento cuantitativo. La elección de los alumnos fue voluntaria; es decir, no estuvo determinado por un grupo de alumnos que tuvieran un alto nivel de conocimiento matemático, pero sí conocían los métodos usuales que requiere un sistema de ecuaciones lineales para ser resuelto. Los 10 alumnos participaron durante cinco sesiones de 2 horas cada una (cinco actividades en total) todos asistieron a las cinco sesiones.

Se debió filmar cada una de las sesiones estipuladas; estas evidencias permitieron comprender mejor los procedimientos descritos por cada grupo de alumnos, ya que ellos, al resolver el problema con el software, verificaban la solución con papel y lápiz, tal como se observa en el siguiente gráfico: Figura 1. Verificación de solución en el papel

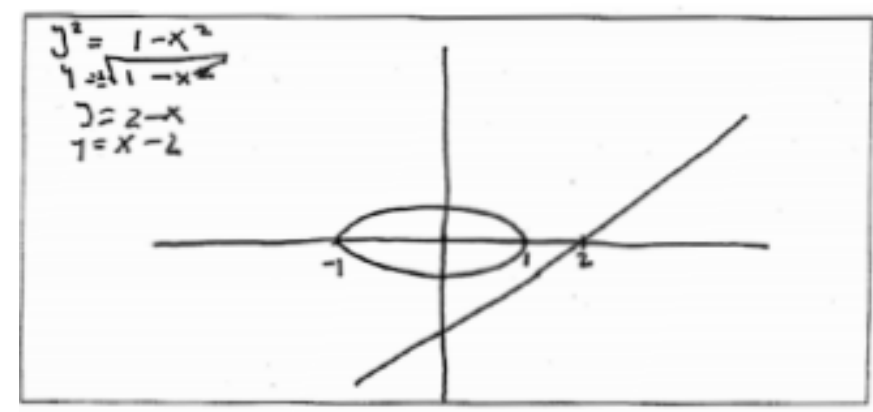

Figura 1. Errores de interpretación gráfica con papel y lápiz.

Como se observa en la figura 1 , se puede constatar que el solo uso de papel y lápiz no es suficiente para dinamizar un problema en términos generales, es importante conocer la génesis instrumental que referencia la importancia del uso de los recursos informáticos en los procesos de enseñanza-aprendizaje de la matemática, de ahí que los alumnos tuvieron la oportunidad de trabajar actividades que les permitieron:

- Razonar sobre el significado del pensamiento geométrico, lógico y variacional de los sistemas de ecuaciones lineales y no lineales, y relacionar las soluciones de estos con el 
significado simbólico y geométrico a partir de la virtualidad, elementos importantes en las clases sincrónicas. Aquí los alumnos logran explorar con el software dinámico, conjeturas relacionadas con el concepto solución de sistemas de ecuaciones lineales y no lineales, en términos generales la relación del software con los tipos de pensamiento matemáticos y sus argumentos, que les permitieron explicar o justificar resultados obtenidos mediante la experimentación como se muestra en la figura 2. Graficando en el papel explican o justifica el resultado obtenido.

\section{Resultados}

Es de interés fundamental describir y analizar en esta investigación el concepto de solución que aportan los alumnos cuando resuelve un problema de matemáticas, mediante el uso de nuevas tecnologías como software dinámico o calculadoras en tiempos del COVID 19. Para llevar a cabo el desarrollo de las actividades y lograr tener evidencias del trabajo de los alumnos, se debió filmar cada una de las sesiones estipuladas; estas evidencias permitieron comprender mejor los procedimientos descritos por cada grupo de alumnos, ya que ellos, al resolver el problema con el software, verificaban la solución con papel y lápiz, tal como se observa en el gráfico: Figura 1. Verificación de solución con papel y lápiz, se referencia la importancia del uso de los recursos informáticos en los procesos de enseñanza-aprendizaje de la matemática, los alumnos tienen la oportunidad de trabajar actividades que les permite: - Razonar sobre el significado geométrico y algebraico de los sistemas de ecuaciones lineales y no lineales y los tipos de pensamiento matemáticos, relacionar las soluciones de estos con el significado simbólico y geométrico.

Aquí los alumnos tienen la oportunidad de explorar, con el software dinámico, conjeturas relacionadas con el concepto solución de sistemas de ecuaciones lineales y no lineales, y utilizar argumentos matemáticos, que les permita explicar o justificar resultados obtenidos mediante la experimentación como se muestra en la figura 2. 


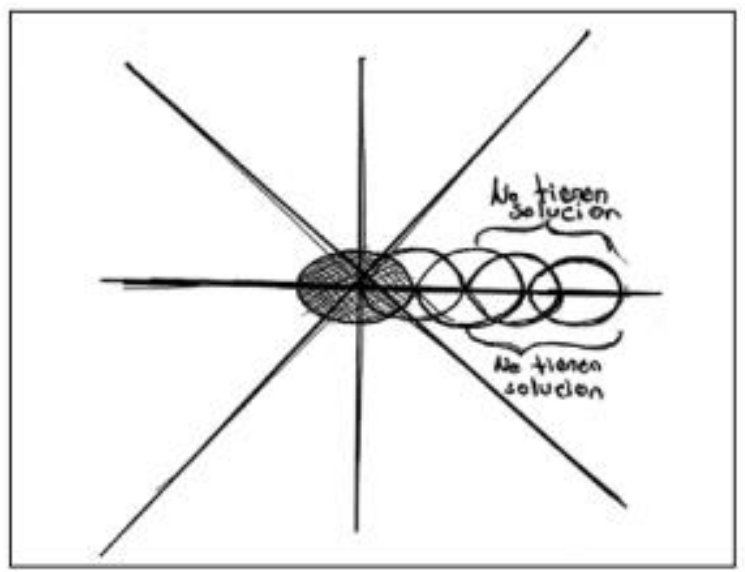

Figura 2. Grafica a partir del uso de la tecnología

Interiorizar una conducta que los llevó a la necesidad de comprobar el concepto de solución, cuando resuelven un sistema de ecuaciones por métodos algebraicos y cuando lo hacen a través del software dinámico, antes de hacer una predicción de un posible resultado que los aleje o los acerque a la solución. Puede suceder que el alumno, usualmente, domine los tres métodos algebraicos para resolver un sistema de ecuaciones lineales, pero al momento de escribir las ecuaciones en la calculadora TI92 sucede que aparece en la pantalla de la CAS una de las cuatro opciones descritas: Figura 3. Resultados obtenidos con calculadora TI-92 (CAS) 1 .

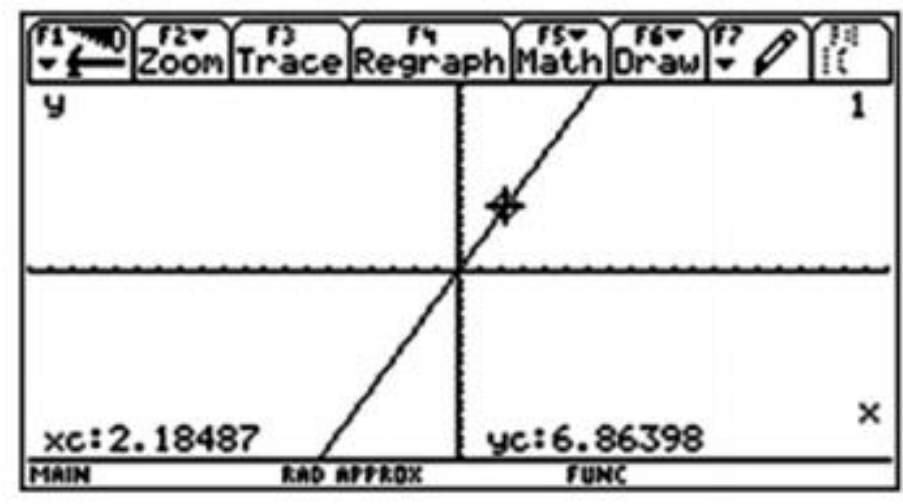

Figura 3. Resultados obtenidos con la Calculadora concepto de Instrumentación.

1. observa las gráficas, pero no el punto de intersección;

2. observa únicamente el plano cartesiano;

3. observa una gráfica;

4. observa el punto de intersección; Aquí, es importante darle al alumno argumentos 
relacionados con los comandos que trae incorporados el software y la función que cumple cada uno de ellos, para que desarrolle estrategias y le facilite una mejor comprensión en el desarrollo de la tarea.

- Usar software dinámico en todas las actividades propuestas, ya que de este aspecto dependió el éxito o el fracaso de los alumnos de este estudio.

- Desarrollar una actitud crítica de los alumnos respecto de los resultados que se obtienen al usar la calculadora y reafirmar el papel fundamental del alumno como elemento racional frente a la automatización y escritura de la misma. Se pretende generar en el alumno una actitud de responsabilidad y coherencia en sus respuestas; es decir, cuestionar por qué él considera que su respuesta es correcta, y no aceptar la primera respuesta automática fuera de contexto que se le pueda ocurrir, para "salir del paso". Esta situación depende del experimentador, quien debe ser cuidadoso en hacer los comentarios pertinentes, y así evitar cualquier mal entendido que pueda perjudicar el proceso de investigación: La falta de autoevaluación en los procesos realizados por un alumno en un contexto de papel y lápiz, puede ser complementada en un ambiente de papel, lápiz y microcomputadora. Esta última, en muchos casos, permite visualizar el error, permitiendo una revisión de su proceso para una mejor aproximación en la resolución de un problema. (Hitt, 2016, p. 42) La incorporación de las tecnologías en el proceso de enseñanza y aprendizaje de las matemáticas no puede dejar fuera el análisis de los diferentes usos que se le pueda dar en esta disciplina, sus ventajas y limitaciones. • Incentivar a los alumnos mediante las actividades que encuentren la solución de problemas a través de los métodos usuales y el uso de los recursos tecnológicos; explicar cómo lo entiende el alumno. El desarrollo tecnológico en las últimas tres décadas no fue asimilado completamente por el sistema educativo. Posiblemente, se deba a la carencia de estudios serios sobre maneras eficientes para utilizar la tecnología en la educación, que nos muestre sus aciertos, así como sus desventajas, y con ello crear una infraestructura de apoyo al profesor de matemáticas para que pueda hacer un buen uso de la tecnología en el aula de matemáticas (Guzmán, et. al., 2012, p. 114). 
Una buena actividad le facilita al alumno desarrollar sus conjeturas, y así mismo ver conexiones que le permiten resolver otros problemas. Hacer matemáticas implica que uno se ocupe de problemas, pero a veces se olvida que resolver un problema no es más que parte del trabajo que se esté llevando a cabo; encontrar buenas preguntas es tan importante como encontrarles soluciones.

- Desarrollar sus habilidades mediante el uso por ejemplo de la calculadora TI-92. La calculadora TI-92 se convierte en un artefacto de ayuda, principalmente, para los alumnos con deficiencias en procedimientos algebraicos. Por ejemplo, cuando resuelven ecuaciones de segundo grado por métodos de factorización confunden la expresión 3x2 $\operatorname{con} 2 \mathrm{x}+\mathrm{x}$.

- Compararlos resultados obtenidos con el software dinámico que trae incorporado la CAS mediante el uso de papel y lápiz.

- Fomentar la actividad de traducción de un problema algebraico a uno de tipo gráfico, con el objeto de hallar soluciones diferentes de un mismo problema. Es posible que para algunos alumnos la calculadora TI-92 les sea completamente desconocida; claro está dependiendo de la escuela donde estudia. Entonces, cuando resuelven un sistema de ecuaciones por métodos algebraicos ya tienen mecanizado el procedimiento para encontrar la solución, y cuando intenten resolverlo con ayuda de la calculadora TI-92, se pueden confundir.

- Desarrollar el trabajo en equipo, de tal manera que cada uno de los integrantes pueda comunicar sus resultados. Es importante señalar que el trabajo en grupo es benéfico, pues la comunicación permite a los alumnos compartir y corregir las tareas que ejecutan, y asimismo tienen la oportunidad de comparar sus resultados. Los puntos señalados anteriormente, “inducen a pensar que los alumnos ubiquen el aprendizaje de las matemáticas, como una actividad útil, que no se reduzca a la memorización de conceptos y procedimientos" Lizarazo (2005 p. 4). Además, con estos puntos se pretende formular preguntas y encontrar relaciones en cada una de las actividades que se vayan a diseñar; por lo que, es posible dar una justificación o prueba de la conjetura, por esta razón en esta investigación fue necesario utilizar los tres niveles de aprendizaje planteados por Pluvinage, F. (2014): 
1. Nivel de entrada, sirve como instrucciones básicas para que el estudiante entre a la actividad tanto en sus aspectos vinculados con el software como en los aspectos matemáticos.

2. Por ejemplo, interacciona con el software aplicando las herramientas del mismo, cuando traza un círculo y coloca un punto sobre un objeto.

3. Nivel de exploración: aquí el estudiante puede identificar relaciones entre objetos y explorar las retroalimentaciones del software. Trabaja a este nivel, por ejemplo, cuando solicita a Cabrí el lugar geométrico de un objeto que se mueve, pero su trayectoria depende de otro.

4. Nivel de estudio matemático. En esta investigación participaron estudiantes del curso de razonamiento cuantitativo. En el periodo de la experimentación todos estaban cursando la asignatura.

No requería que los alumnos tuvieran un alto nivel de conocimiento matemático, pero sí conocían los métodos usuales que requiere un sistema de ecuaciones lineales para ser resuelto.

Los 10 alumnos participaron durante cinco sesiones de dos horas cada una (cinco actividades en total) todos asistieron a las cinco sesiones. Las dos primeras actividades fueron abordadas individualmente y las otras tres en grupos de tres alumnos en este último el estudiante, a partir de la observación y con la comparación se puede formular conjeturas y validarlas matemáticamente, para cual se explicita lo siguiente: En un primer ensayo cuando abordaron el problema de la pista de carreras (problema 12., P. 91) del texto guía, se pudo observar que el estudiante únicamente hace uso de papel y lápiz los cuales se limitan a recordar fórmulas para tratar de modelar el problema a una ecuación de primer o segundo grado y llegar a la respuesta lo más rápidamente posible.

Schoenfeld (1987), afirma que: "una hipótesis básica consiste, en que, a pesar de su complejidad, las estructuras mentales de los alumnos pueden ser comprendidas y tal comprensión ayudará a conocer mejor los modos en que el pensamiento y el aprendizaje tienen lugar" (p. 420). Si estas estructuras se refuerzan con otros recursos tales como: un 
software de geometría dinámica, pueden llegar a ser herramientas interesantes para los alumnos en el proceso de aprendizaje de las matemáticas. Propuesta implementada La propuesta de los estándares del NCTM (2018) enuncia varios procesos del quehacer matemático como ejes de la propuesta curricular, para que los docentes la consideren y les permita mejorar los procesos de aprendizaje en los alumnos del nivel medio superior (p. 53).

Ahora bien, ¿por qué se cree que pueden producirse cambios en la forma de enseñar y aprender matemáticas con las nuevas tecnologías intelectuales principalmente con la CAS? La respuesta se fundamenta en los sistemas de representación que ofrecen estas tecnologías: dinámicos y con la posibilidad de establecer una mejor correspondencia entre el universo visual y el numérico (López, 2003, p. 6).

Este tipo de análisis no proporciona una base adecuada para el estudio de los sistemas de ecuaciones lineales y no lineales mediante el uso de la CAS, ya que no se percibe la conexión entre los métodos tradicionales y el concepto solución, que puedan dar los alumnos con la CAS y la relación que existe con lápiz y papel; sin embargo, es considerada para motivar la investigación en la recolección de datos. Se les presentó el siguiente problema a los estudiantes tomados del texto guía. La figura 4, muestra una pista de carreras donde la parte interior consiste de un rectángulo que es dos veces más largo que el ancho y de dos semicírculos.

La pista es de 7 metros de ancho y el tartán que se necesitó para recubrirla fue de 1354 metros cuadrados. Encuentra las medidas del radio interior de los semicírculos. Para facilitar las cuentas se toma la aproximación $p=22 / 7$ Figura 4. Esquema de una pista de carreras, para un problema matemático propuesto 2 National Council of Teacher of Mathematics 108

La influencia de avances en la tecnología para el aprendizaje del estudiante de ingeniería en la solución de problemas 1. Procedimiento algebraico Como piden calcular el radio r, se tiene entonces que los lados del rectángulo son 2r y 4r. Por lo tanto el área de la pista cubierta por el tartán es: $4 \mathrm{r}(2 \mathrm{r}+14)+\mathrm{p} .(\mathrm{r}+7) 24 \mathrm{r}(2 \mathrm{r}+14)+\mathrm{p} .(\mathrm{r}+7) 2-(4 \mathrm{r}(2 \mathrm{r})+\mathrm{pr} 2)=13548 \mathrm{r} 2+$ $56 \mathrm{r}+\mathrm{p} \mathrm{r} 2+14 \mathrm{p} \mathrm{r}+49 \mathrm{p}-8 \mathrm{r} 2=135456 \mathrm{r}+14 \mathrm{pr}+49 \mathrm{p}=1354$ se remplaza p por 22/7 56r + $14(22 / 7) \mathrm{r}+49(22 / 7)=1354$. Así que:100r $+154=1354100 \mathrm{r}-154=1354-154$ 100r $=$ 
1200 dividiendo la ecuación entre 100 se tiene entonces que $r=12 \mathrm{~m} 2$. Procedimiento para construir la pista de carreras con software dinámico Con este procedimiento se pretende mostrar una forma de construir la pista de carreras el cual permite al estudiante ir analizando cada paso para llegar a una posible solución:

1. Trace un segmento de recta y sobre dicho segmento ubique un punto sobre objeto P.

2. Calcule la longitud del segmento desde el extremo A hasta el punto sobre objeto P. Con transferencia de medidas traslade la longitud del segmento $\mathrm{AB}$, y con centro en uno de los extremos trace una circunferencia, de tal manera que el radio de la circunferencia sea la longitud del segmento AP, podrás analizar que cuando mueve el punto $\mathrm{P}$ el radio varía.4.

Ahora trace una recta que pase por el centro de la circunferencia y con punto de intersección construya el ancho del rectángulo 2r. Para construir el largo del rectángulo trace dos circunferencias hagan centro en cualquiera de los extremos, y para trazar el ancho del rectángulo encuentre los puntos de intersección de la perpendicular con la circunferencia.

Con un procedimiento análogo, se construye el otro extremo de la pista, trazando arcos y ocultando las circunferencias y las rectas perpendiculares entre otros elementos. El ancho se traza con edición numérica y transferencias de medidas, es importante, trazar los círculos para encontrar los puntos de intersección donde se pueda ver la variedad del ancho de la pista, se hace algunos procedimientos aritméticos sobre la diferencia de las áreas, tanto de los círculos como de los rectángulos; y así la pista queda funcionando perfectamente. 4. Construcción, Figura 5; Figura 6; Figura 7. Parte de la construcción solución Figura 8. 


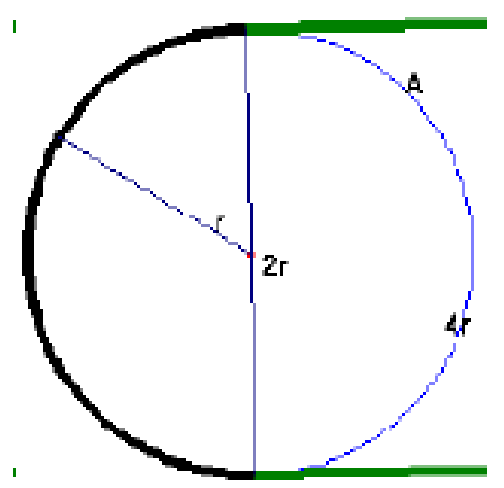

Figura 3.

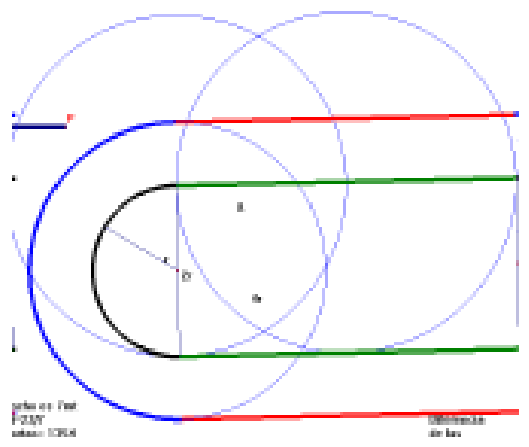

Figura 4.

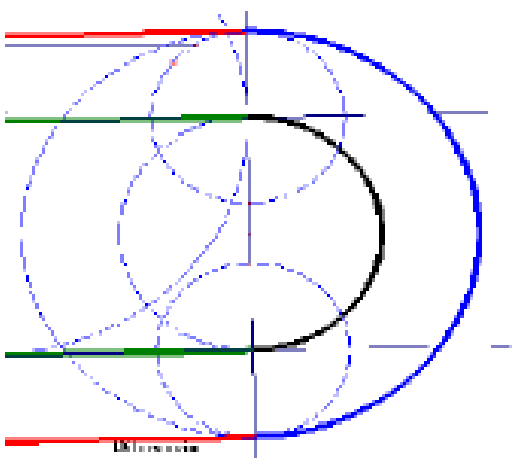




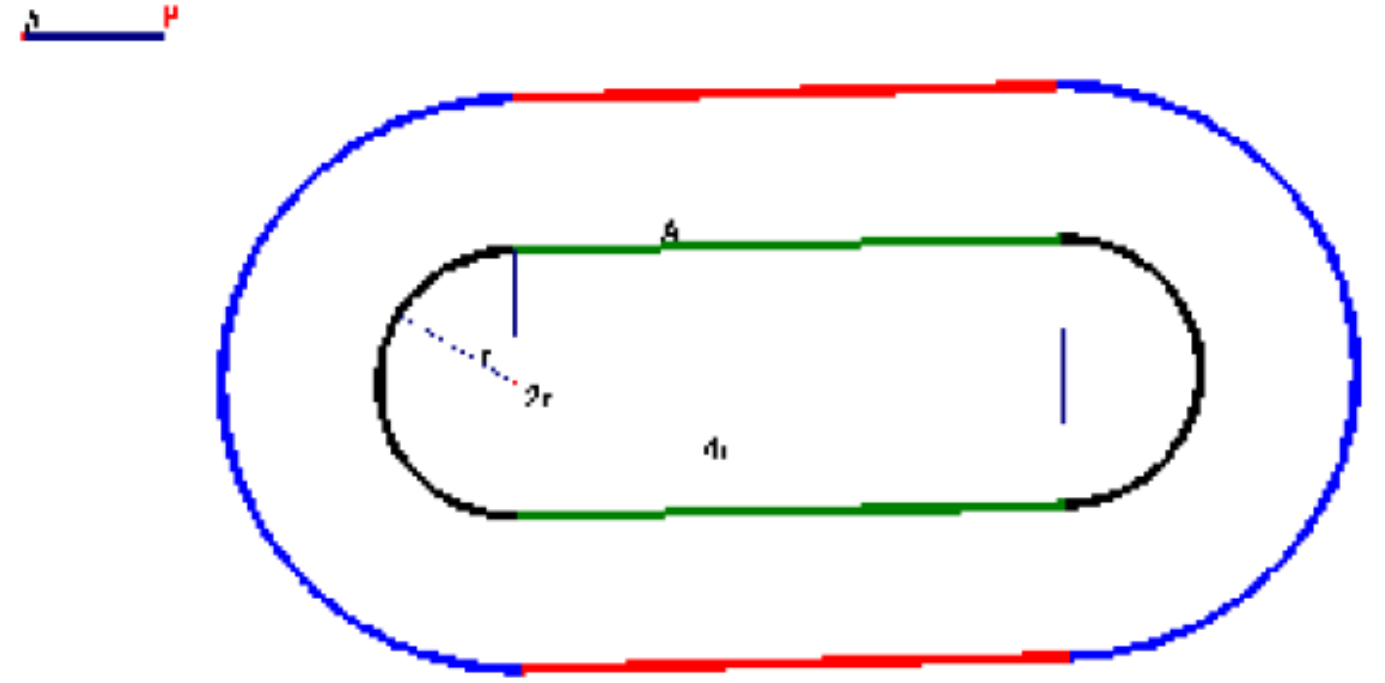

Figura 4. Construcción a partir de los razonamientos críticos y los tipos de pensamiento.

\subsection{Funcionamiento del problema}

Para conocer el ancho de la pista haga clic dos veces en A y podrás ver como el problema se dinamiza y se puede visualizar todos los elementos que intervienen en el mismo. Movería el ancho de la pista para $\mathrm{N}=0,0.1,0.2$, $\mathrm{n}$ metros De igual forma el área de la pista varia moviendo el punto $\mathrm{P}$, en función del radio, pues tanto el área de los rectángulos como de los dos semicírculos dependen de que tan grande o pequeño sea r. Según Bosch, el programa de matemáticas está orientado en la resolución de problemas. La habilidad de los estudiantes para razonar, resolver problemas y emplear las matemáticas para comunicar ideas sólo podrá ser desarrollada, si los estudiantes participan activa y frecuentemente en estos procesos. Para Pluvinage (2003) es importante hablar de los niveles de aprendizaje a través de la computación sobre objetos matemáticos y conceptos. 7. Preguntas 1. ¿Qué sucede en el problema si varía el ancho de 7 a 225 metros en la pista ¿se puede afirmar que el radio es 6 metros? 2. ¿Suponga que desconoces el ancho de la pista y está cubierta por 1354, 1300 y 
1250 metros, si el radio es de 12 metros se puede esperar que estos valores no afecten el ancho de la pista? Explique gráfica y analíticamente.3. Encuentre una ecuación general para un ancho cualquiera que resuelva cualquier área de una pista de carreras.

\section{Conclusión}

El empleo del enfoque que plantea Pluvinage en cada actividad, para realizarlas en un curso como "La computadora en el aprendizaje de las matemáticas" resultó ser pieza fundamental para poder aplicar los tres niveles de aprendizaje en la resolución de problemas matemáticos, como fueron: Nivel de entrada, Nivel de Exploración y Nivel de estudio matemático. Y como este tipo de análisis si proporciona una base adecuada para el estudio de los sistemas de ecuaciones lineales y no lineales mediante los sistemas de representación que ofrecen estas tecnologías, como herramientas que aportan al aprendizaje con una dinámica que posibilita el establecer una mejor correspondencia didáctica entre el universo visual y el numérico (López, 2013, p. 6).

En recientes foros internacionales relacionados con la virtualidad en tiempos de pandemia, mucho docente se pregunta, ¿qué hasta cuándo van dejar da dar las clases virtuales? Por la complejidad de tener que manejar las plataformas diseñadas para facilitar la contaminación e improvisación que en muchas ocasiones se llega al aula. Para Morris Kline repetir ruidos correctos. La respuesta es muy sencilla, Japón y muchos países desarrollados llevan más de 30 años impulsando el aprendizaje autónomo donde el maestro cumple la función de guía para guiar procesos a través de la virtualidad, pero esto requiere y exige preparación de alto nivel. 


\section{Referencias Bibliográficas}

Artigue, M. (2016). Etude de la dynamique d'une situation de classe : Une approche de La reproductibilité. Recherches en Didactique des Mathématiques. Vol.7 (1), pp. 5-62

Artigue, M. (2001). Learning Mathematics in CAS Environment: The genesis of a reflection about instrumentation and the dialectics between technical and conceptual work, CAME 2001, FreudenthalInstitut, Utrecht http://1tsn.mathstore. ac.uk/came/events/freudenthal/theme1.html.

Artigue, M. (2002). Learning Mathematics in CAS environment: The genesis of a reflection about instrumentation and the dialectics between technical and conceptual work. International Journal of Computes for Mathematical Learning, 7, 245-274.

Brahier, D. (2000). The Role of Graphing Calculators in Advancing Discourse. Focus on Learning Problems in Mathematics (22) 3 y 4, pp. 80-92, summer and Fall Editions

Balacheff, N. and Kaput, J. (1996). Computer Based Learning Environment in Mathematics, In Bishop, A, J. et al, International Handbook of Mathematical Education pp. 469501. Netherlands: Kluwer Academic Publishers. Chevallard, Y. (1999). L'analyse des pratiques enseignantes en théorie anthropologique du didactique [The analysis of teaching practices in didactical anthropological theory]. Recherches en Didactique des Mathématiques, 19, 221-266.

Engler, A. et al. (2011). Propuesta didáctica para estudiar sistemas de ecuaciones lineales. sondeo de opiniones. Educación Matemática vol. 13, No. 2, pp. 127-129.

Guin, D. and Trouche, L. (2012). The complex process of converting tools into mathematical instruments: the case of calculators. Int. Jour. Of Computers for Math. Learnin 3, pp. 195-227. Guin and Trouche (2012). Mastering by the teacher of the instrumental genesis in CAS environments: necessity of instrumental orchestrations. ZDM, vol. 34 (5). Recuperado de: http:// www.fizkarlsruhe.de/fiz/publications/zdm/zdm025a4.pdf

Guzmán, J. y Kieran, C. (2002). The Role of Calculation InstrumentalGenesis:theCase of Nicolas and Factors andDivisors. In Proceedings of the 26th Annual Cpnference, Anne D. Cpckburn and Elenena Nardi (Eds.). Norwich. pp. 41-48.

Guzmán, J. et al. (2012). El Currículo de Matemáticas en México en la Escuela Media. En A. Maz, M. Torralbo y C. Abraira 110 La influencia de avances en la tecnología 
para el aprendizaje del estudiante de ingeniería en la solución de problemas (Eds), Currículo y Matemáticas en la Enseñanza Secundaria en Iberoamérica, p. 114. Córdoba, México: Universidad de Córdoba.

Grassl, R. and Mingus, T. (2012). On the shoulders of technology: calculators as cognitive amplifiers. Int. J. Math. Educ. Sci. Technology., (33) 5, pp. 715-723.

Hitt, F. (1996). Educación Matemática y uso de herramientas tecnológicas. En M. Santos y E. Sánchez (Eds). Perspectiva en Educación Matemática, pp. 21- 44. México: Grupo Editorial Iberoamérica.

Hong, and Thomas, M. (2014). Building Newton Raphson Concepts with CAS. PME26 Inglaterra, pp. 105-112. Horgan, J. (1993). The death of Proof. Scientific American 269, p. 47. Kaput, J. (1996). Computer - Learning Environment in Mathematical en Bishop, A. J. et al, International Handbook of Mathematical Education, pp. 515556. New York, USA: Macmillan.

Kieran, C. y Guzmán, J. (2013). The Spontaneous Emergente of Elementary NumberTheoretic Concepts and Techniques in Interaction with Computing Technology. In Proceedings of the 2013 Joint Meetinf of PME an PMENA, Neil A. Paterman, Barbara J. Doughert and Joseph Zilliox (Eds) vol. 3, pp, 141-148.

Lagrange, J. B. (2013). Learning tecniques and concepts using CAS:Apractical and theoreticalreflection. " In. J. T. Fey (Ed.), Computer Algebra Systems in Secondary school mathematics education (pp. 269-283). Reston, VA: National Council of Teachers of Mathematics

López, L., (2003). Construyendo un camino de la conjetura a la organización deductiva de la información mediante la exploración con la calculadora TI-92 plus. Tesis de Maestría no publicada. Departamento de Matemática Educativa, Cinvestav-IPN. México

Moreno, L. y Santos, M. (2011). De la herramienta al instrumento una perspectiva informática. Educación Matemática vol. 13, No. 2, p. 83.

Moreno, L. and Block, D. (2012). Democratic Access to Powerful Mathematics. En Lyn, D. et al, Handbook of International Research in Mathematics Education, pp. 307-318, Kluwer Academic Publishers. Mariotti, M. (2002). The Influence of Technological Advances on Students' Mathematics Learning. International Research

Mariotti, M. A. (2013). Influence of technology advances on students`s math learning. In L. English, Handbook of Inter. Research in Math. Educ., Mahwah, NJ: Lawrence Erlbaum. London. P. 707. 
National Council of Teachers of Mathematics (2014). Curriculum and evaluation Standers for School Mathematics. Reston, VA: NCTM. USA. Oaxaca, J. (2014). El papel que desempeña la calculadora en la adquisición de conceptos matemáticos en alumnos de segundo grado de secundaria. Tesis de Maestría no publicada. Departamento de Matemática Educativa, Cinvestav-IPN. México.

Ramírez, M. (2017). El uso de la calculadora graficadora y la resolución de problemas algebraico-verbales, en el estudio de sistemas de ecuaciones lineales con dos incógnitas. Tesis de Maestría no publicada. Departamento de Matemática Educativa Cinvestav-IPN. México.

Rubenstein, R. (2012). Teaching the Line of Best Fit with a Graphing Calculator. Calculators in Mathematics Education. Yearbook, NCTM. USA, p. 5

Páez,C.(2014).Formas de razonamiento que exhibenestudiantes de preparatoria en ambientes de resolución de problemas con el uso de software dinámico. Tesis de Maestría no publicada. Departamento de Matemática Educativa, Cinvestav-IPN. México.

Santos, M. (1998). Instructional qualities of a successful mathematical problem solving class. International Journal of Mathematical in Science and Technology, p. 631.

Santos, M. (2002). La naturaleza de las matemáticas y sus implicaciones didácticas, Revista perspectiva pp. 420- 421. Departamento de Matemática Educativa, Cinvestav-IPN. México.

Stewart, I. (2009). Change. En L. Steen (Ed), On the Shoulders of Giants. New Aproache to Numeracy, pp. 180-217. Washington, DC, USA: National Academy Press. Verillon, p. and Rabardel, P., (1995). Cognition and artifacts: A contribution to the study of the thought in relation to instrumented activity. European Journal of Psychology of education, X, pp. 77-101. Ward, R. (2000). Observing High School Students Strategies and Misconceptions as They Use Graphing Calculators. Focus on Learning Problems in Mathematics (22) 3 y 4, pp. 28-39, summer and Fall Editi 